\title{
Impacto de los servicios de agua y saneamiento sobre las enfermedades diarreicas en los niños de la sierra del Perú.
}

\author{
Impact of water and sanitation services on diarrheal \\ diseases in children in the highlands of Peru.
}

\author{
Carmen Vanessa Montero Contreras ${ }^{1}$
}

\begin{abstract}
Drinking water is important for human life. Goal 6 of the SDGs indicates ensuring the availability and sustainable management of water and sanitation and good water quality for all. Lack of water resources, low water quality and inadequate sanitation affect food security and people's health. In this sense, diarrhea is one of the diseases caused by the lack of drinking water. According to the WHO, diarrhea is the second leading cause of death for children under the age of five. Peru ranks eight in the world among the countries with the largest water reserve, however, only $1 \%$ of the total available is consumed. At the end of $2019,9.2 \%$ of the population did not have access to the drinking water network and $25.1 \%$ did not have access to sewerage services. This situation is exacerbated in urban and rural areas of the country, the latter being the one that presents the greatest gap in access to water and sewerage. The prevalence of diarrhea in girls and boys under five years of age in rural areas was $12.5 \%$ in 2019 , according to the INEI. The study evaluates the impact of water and sewerage services in the highlands of Peru on acute diarrheal diseases in children for 2019, using the Propensity Score Matching (PSM) technique. It is obtained that accessing one of these services decreases the probability of presenting diarrhea by $3.7 \mathrm{pp}$.
\end{abstract}

Keywords: Drinking water, diarrhea, impact assessment, matching, sanitation

\section{RESUMEN}

El agua potable es importante para la vida humana. El objetivo 6 de los ODS indica garantizar la disponibilidad y la gestión sostenible del agua y el saneamiento y buena calidad del agua para todos. La falta del recurso hídrico, la baja calidad del agua y un inadecuado saneamiento afecta a la seguridad alimentaria y a la salud de las personas. En ese sentido, la diarrea es una de las enfermedades producidas por la falta de agua potable. Según la OMS (2017), la diarrea son la segunda mayor causa de muerte de niños menores de cinco años. Perú ocupa el puesto ocho a nivel mundial de los países con mayor reserva hídrica, sin embargo, sólo el $1 \%$ del total disponible es consumido. A fines del 2019 , un $9,2 \%$ de la población no tuvo acceso a la red de agua potable y un $25,1 \%$ sin alcantarillado. Esta situación se agudiza en las zonas urbanas y rurales del país, siendo ésta última la que presenta una mayor brecha en el acceso al agua y alcantarillado. La prevalencia de diarrea en niñas y niños menores de cinco años en el área rural fue de $12.5 \%$ en 2019, según el INEI. El estudio evalúa el impacto de los servicios de agua y de alcantarillado en la Sierra del Perú sobre las enfermedades diarreicas agudas en niños para el 2019, usando la técnica de Propensity Score Matching (PSM). Se obtiene que acceder a uno de estos servicios disminuye la probabilidad de presentar diarrea en 3.7 pp.

Palabras clave: Agua potable, diarrea, evaluación de impacto, emparejamiento, saneamiento

Received: 16 de noviembre del 2021

Accepted: 10 de diciembre del 2021

\section{Introducción}

El agua es uno de los motores del desarrollo sostenible y base para la producción de alimentos y de la energía, impulsa el desarrollo socioeconómico y es indispensable para la vida de los seres humanos. En julio del 2010, la Asamblea General de las Naciones Unidas reconoció el derecho humano al agua y al saneamiento. Según Naciones Unidas, las personas deben tener acceso a una cantidad de agua suficiente (UNESCO, 2019) para el uso personal y doméstico. De acuerdo con la Organización Mundial de la Salud (OMS), son necesarios entre 50 y 100 litros de agua por persona y día para cubrir las necesidades más básicas (beber, cocinar y mantenerse limpios). Así también, las Naciones Unidas indica que el agua debe ser saludable (la mala calidad del agua y un saneamiento inadecuado influyen en la prevalencia de enfermedades diarreicas agudas) y físicamente accesible (según la OMS, la fuente de agua debe ubicarse a menos de $1 \mathrm{~km}$ de la vivienda y el tiempo de desplazamiento para el recojo no debe ser mayor a los 30 minutos).

En el objetivo 6 de los Objetivos de Desarrollo Sostenible (ODS) se indica garantizar la disponibilidad de agua y su gestión sostenible y el saneamiento para todos. La falta del recurso hídrico, la baja calidad del agua y un deficiente saneamiento afecta a la seguridad alimentaria y a la salud de

${ }^{1}$ Pontificia Universidad Católica del Perú. E-mail:carmen.montero@pucp.edu.pe

How to cite: Montero Contreras, C. V. (2022). Impacto de los Servicios de Agua y Saneamiento sobre las enfermedades diarreicas em los niños de la Sierra del Perú. ANÁLISIS ECONÓMICO Y FINANCIERO, 5(1), 1-8. https://doi.org/10.24265/raef.2022.v5n1.45 
las personas. En el 2020 una de cada cuatro personas carecía de una fuente de agua potable gestionada de forma segura y casi la mitad de la población mundial no tenía acceso a servicios de saneamiento adecuados, según la Organización Mundial de la Salud (OMS, 2021). En el mismo informe, se indica que al inicio de la pandemia de Covid-19, tres de cada 10 personas en el mundo no tuvieron acceso a lavarse las manos con agua y jabón en sus hogares. Existe una brecha en el sistema de servicio de acceso a agua y alcantarillado entre los países desarrollados y los países en desarrollo, ya que los primeros cuentan con un buen sistema de abastecimiento de agua potable y salubre, mientras que para la mayoría de los países en desarrollo ello no ocurre, como es el caso peruano.

El agua limpia y saneamiento son dos factores para evitar la propagación de enfermedades infecciosas y parasitarias (González,Velázquez \& Epele (2013). Los niños son el grupo más vulnerable porque su sistema inmunológico está en desarrollo y por la falta de información en prevención de enfermedades. Según la OMS, la diarrea ocupa el segundo lugar en las causas de muerte de niños menores de cinco años (OMS, 2017). De modo que, cada año se producen 1 700 millones de casos de enfermedades diarreicas infantiles a nivel mundial y 525000 niños menores de cinco años fallecen. Perú ocupa el puesto ocho a nivel mundial, del país con mayor reserva hídrica, sin embargo, sólo el $1 \%$ del total disponible es consumido. A fines del 2019, un $9,2 \%$ de la población no tuvo acceso a la red de agua potable y un $25,1 \%$ sin instalaciones de desague. Así también existe grandes brechas entre las zonas urbanas y rurales de Perú, siendo ésta última la que tiene una mayor brecha entre acceso al agua y a alcantarillado. Según el Ministerio de Vivienda, Construcción y Saneamiento, en el 2019 , el $40.1 \%$ y el $18.3 \%$ de la población rural y población urbana respectivamente no tuvieron acceso al servicio de agua potable (MVSC, 2021a). Y datos de las enfermedades diarreicas agudas (EDA), en el 2019, la ocurrencia de diarrea en niñas y niños menores de cinco años fue $11.5 \%$ (INEI, 2020a). Además, por áreas urbana y rural fue de $11.1 \%$ y $12.5 \%$ respectivamente. $\mathrm{Y}$ a nivel de sexo, fue de $12.7 \%$ en niños y $10.2 \%$ en niñas. Así, el presente trabajo tiene como principal objetivo evaluar el impacto de los servicios de acceso a agua y a alcantarillado en la región Sierra del Perú en la prevalencia de enfermedades diarreicas agudas (EDA) en los niños menores de cinco años. Para ello, se utiliza la técnica de Propensity Score Matching (PSM), la cual fue publicada por primera vez por Paul Rosenbaum y Donald Rubin en 1983. La estructura del documento inicia con una breve introducción, luego se presenta una revisión de la literatura, así como la descripción de los datos y la metodología. En la tercera sección, los resultados de la evaluación de impacto. Y en la última sección, se presentan algunas conclusiones del estudio.

\section{Revisión literaria y Metodología}

El Ministerio de Vivienda, Construcción y Saneamiento (MVCS, 2021a), realizó un diagnóstico de la situación de brechas de infraestructura o de accesos a servicios públicos del sector vivienda, construcción y saneamiento. Para el servicio de agua potable, se presentó dos indicadores brecha para las zonas rurales. El primer indicador(cobertura) fue el porcentaje de la población rural sin acceso al servicio de agua potable mediante red o pileta públicas y el segundo, porcentaje de viviendas con servicio de agua con cloro residual menor al límite permisible $(0.5 \mathrm{mg} / \mathrm{l})$. Y para el servicio de alcantarillado, el indicador fue el porcentaje de la población rural sin acceso al servicio de alcantarillado u otras formas de disposición sanitaria de excretas. Según datos del INEI, en el 2019, la población rural ascendió a 7 605868 habitantes, de los cuales el 59.9\% contó con acceso al servicio de agua potable y el $40.1 \%$ no cuenta con acceso a este servicio. En relación al segundo indicador que mida la calidad (se considera una muestra no satisfactoria cuando la concentración de cloro residual es menor a $0.5 \mathrm{mg} / \mathrm{l}$ ), datos de la Encuesta Nacional de Programas Presupuestales (ENAPRES) 2019, indica que, del total de viviendas de la zona rural ( 2069678 ), el 3.8\% se beneficiaron con servicios de agua potable con presencia de cloro residual mayor al límite permisible, mientras que el $96.19 \%$ de las viviendas mostraron una concentración de cloro residual inferior $\mathrm{o}$ igual a $0.5 \mathrm{mg} / \mathrm{l}$. El indicador de cobertura de servicio de alcantarillado, del total de la población rural de 7605868 habitantes (2019), sólo el $42.7 \%$ contó con acceso al servicio de alcantarillado y el $57.29 \%$ no tuvo acceso a este servicio.

Tabla 1

En el 2019, los departamentos que presentaron una brecha del servicio de acceso a agua potable superior del promedio nacional (41\%) fueron Loreto (80,4\%), Ucayali (71,5\%), Madre de Dios (60,33\%), Puno (57,2\%), Tacna (56,8\%), Junín (52\%), Moquegua (49\%), Pasco (48.79\%), Huánuco $(46.52 \%)$ y Piura $(46.34 \%)$.

\section{Tabla 2}

González, Velázquez \& Epele (2013), cuantifican el efecto de contar con servicios de alcantarillado sobre la incidencia de la diarrea en niños menores de 24 meses en Argentina. Para ello, usó la Encuesta Nacional de Nutrición y Salud (ENNyS) 2004-2005 y aplicó la metodología de diferencias simples (grupo de tratamiento versus grupo de comparación) con la técnica de propensity score matching. Entre los resultados obtenidos, concluye en cuanto al efecto promedio (ATE), que los niños que residen en una vivienda con cobertura de alcantarillado o cámara séptica y pozo ciego reducen hasta 6 puntos porcentuales de la probabilidad de tener diarrea. tienen menor probabilidad de sufrir episodios de diarrea (entre 3 y 7 puntos porcentuales).

BID (2021), realizó un estudio para evaluar el efecto de un programa de agua potable y saneamiento en comunidades rurales en cuatro departamentos de Bolivia (Chuquisaca, La Paz, Cochabamba y Potosí). Se usó como fuente de datos una encuesta de seguimiento intermedia y un diseño de evaluación experimental por el cual las comunidades fueron seleccionadas aleatoriamente a los grupos de tratamiento y de control. Se obtuvo que, el Programa, frente al grupo de control, aumentó en 47.2 y 72.3 puntos porcentuales (pp.) el uso de fuentes mejoradas de agua e instalaciones de saneamiento respectivamente. En cuanto a la salud, el Programa disminuyó la prevalencia de diarrea en 10.4 pp frente al grupo de control, tanto en niños y niñas entre 6 y 17 años como en adultos hombres entre 18 y 49 años. No se obtuvo un efecto estadísticamente significativo en la reducción de diarrea en menores de 0 a 5 años, ni en mujeres adultas. 
MVCS (2021b), determina el impacto del Programa Nacional de Saneamiento Urbano (PNSU) en desnutrición crónica, enfermedades diarreicas agudas y mortalidad en niños menores de cinco años. Los datos usados fueron las bases administrativas del Ministerio de Salud y el Sistema Integrado de Proyectos del MVCS para los años 2017 al 2019. Se empleó las regresiones multivariadas con efectos fijos distritales, con las cuales concluye que existe una relación negativa entre el aumento de proyectos terminados de agua y de saneamiento del PNSU y las enfermedades diarreicas agudas, desnutrición crónica infantil y mortalidad infantil, sin embargo, la única estadísticamente significativamente a niveles convencionales (nivel de confianza del 90\%). De modo que, un incremento de siete proyectos del PNSU disminuye un caso de EDA por cada 100 habitantes en el distrito.

Lara y García (2019) evalúan los efectos en la salud de los hogares del Valle del Mezquital (México) relacionadas a la falta de los servicios de agua potable, saneamiento y el uso de aguas contaminadas para tareas domésticas. Se usó los datos obtenidos de la Encuesta Nacional de Salud y Nutrición (ENSANUT) del 2012, publicada por el Instituto Nacional de Salud Pública (INSP) bajo un modelo logístico binomial que incluyó variables sociodemográficas y otras relacionadas con los servicios de acceso a agua potable y saneamiento. El estudio concluyó que, la presencia de enfermedades gastrointestinales se encuentra asociada al tipo de abastecimiento y a su frecuencia. El poder explicativo del modelo fue del $86 \%$.

Carrasco (2013), cuantifica el impacto del consumo de agua potable sobre la salud. Utilizó la técnica de Propensity Score Matching (PSM). Los datos que se usaron fueron de la Encuesta Demográfica y de Salud Familiar (ENDES)2007, realizada por el INEI. Sus resultados muestran si un hogar tiene acceso al agua potable disminuye la prevalencia de diarrea en promedio $4.8 \%$. Los rangos del impacto se encuentran entre $4.4 \%$ (con Kernel bandwidth 0.02 ) a $5.5 \%$ (5-Vecinos cercanos).

Marino (2020), evalúa el impacto de los servicios públicos de agua potable y alcantarillado sobre los logros educativos en el Perú, donde usa la técnica diferencias en diferencias, concluye que para los estudiantes de segundo grado de primaria, los servicios de saneamiento en zonas rurales suben las notas estandarizadas de matemática y comprensión lectora en 0.07 y 0.056 desviaciones estándar respectivamente, así también que, las niñas son las más beneficiadas del acceso a los servicios de saneamiento.

Sotelo (2016), examina el impacto que en el Perú tuvo el acceso a los servicios de agua y saneamiento sobre la desnutrición crónica infantil (DCI), se usó la técnica del Propensity Score Matching y la base de datos de la Encuesta Demográfica y de Salud Familiar (ENDES) de los años 2010 y 2015. En la evaluación de impacto de acceso al agua, se encuentra que, en el 2015, ésta disminuye la incidencia de desnutrición en $1.83 \%$. Y el acceso al saneamiento, contribuye a una reducción en la probabilidad de padecer $\mathrm{DCl}$ a $7.10 \%$. De los dos, el que tiene un mayor nivel de incidencia en la diminución de la $\mathrm{DCl}$ es el servicio de saneamiento, tanto para el $2015(-7.10 \%)$ como para el 2010(-9.88\%). Mientras que, el servicio de agua potable tuvo un impacto de $-1.83 \%$ y $-2.33 \%$ para los años 2015 y
2010 respectivamente. La investigación, también concluye que la mayor incidencia se da en el sector rural respecto al urbano.

Para evaluar el impacto del acceso del agua y del alcantarillado sobre la probabilidad de tener diarrea, se hace necesario saber el resultado sobre los beneficiarios del tratamiento si es que no hubiesen recibido acceso al agua, al alcantarillado o ambos. También, conocer el efecto sobre los que no tienen acceso al agua potable ni al alcantarillado si es que fuesen beneficiarios de ésta. En la realidad no se puede conocer dichos efectos, por lo que, las técnicas de evaluación de impacto, nos permite realizar dichos escenarios. En el estudio se usa la técnica del Propensity Score Matching (PSM) para poder comparar a los niños que cuentan con alguno de los servicios (agua potable, alcantarillado o ambos, denominados grupo de tratamiento) respecto a los que no lo tienen (grupo de control). Se hace necesario que aquellos que no cuenten con estos servicios sean muy similares en algunas características al grupo de tratamiento. El PSM es una técnica cuasiexperimental y fue introducida por primera vez con el trabajo de Rosembaum y Rubin(1983) que resolvieron la maldición de las dimensiones, ya que se resuelve el problema de la dimensionalidad al resumir toda la información de múltiples variadas en un sola. Esto se denomina \puntuación de propensión", el cual es un número real entre 0 y 1 (probabilidad) Con esta técnica, se busca el soporte común, donde el grupo de tratamiento y el no tratado tienen características muy similares. Luego que se tiene el soporte común, se hace el emparejamiento, para ello se puede usar por el vecino más cercano, por estratificación, emparejamiento de radio o el emparejamiento de Kernel. Para realizar el PSM, se debe cumplir dos supuestos:

a) Independencia condicional: Dado un conjunto de variables $\mathrm{X}$ observadas no afectas por el tratamiento, los resultados de $\mathrm{Y}$ son independientes de la asignación del tratamiento.

$$
\begin{gathered}
A T E \rightarrow\left(Y_{I}^{T}, Y_{i}^{C}\right) \perp T_{i} \mid X_{i} \\
A T E T \rightarrow Y_{i}^{C} \perp T_{i} \mid X_{i}
\end{gathered}
$$

Donde ATE es el efecto medio del tratamiento, average treatment effect $y$ ATET es el efecto medio del tratamiento sobre los tratados, average treatment effect on the treated.

b) Soporte común: Las observaciones del tratamiento tienen observaciones de comparación cerca de la distribución del PSM.

Efectos del tratamiento:

$$
\begin{gathered}
\operatorname{ATET}_{P S M} \rightarrow E_{P(X) \mid T=1}\left\{E\left[Y^{t} \mid T=1, P(X)\right]-E\left[Y^{C} \mid T=0, P(X)\right]\right\} \\
\operatorname{ATET}_{P S M} \rightarrow \frac{1}{N_{T}}\left[\sum_{i \in T} Y_{i}^{T}-\sum_{j \in C} w(i, j) \cdot Y_{j}^{c}\right]
\end{gathered}
$$

\section{Resultados y discusión}

Para analizar el impacto del acceso al agua potable y alcantarillado sobre la disminución de enfermedades diarreicas agudas en niños menores de cinco años en la sierra del Perú se usó la base de datos de la Encuesta 
Demográfica y de Salud Familiar (ENDES) del año 2019 que aplica el Instituto Nacional de Estadística e Informática (INEI). Esta encuesta recoge información sobre la salud materna e infantil, atención al embarazo y parto, es decir, tendencias determinantes de la fecundidad, mortalidad y salud. Así también incluye mecanismos para poder prevenir el SIDA y las agresiones físicas en el hogar.

a) Variable resultado:

Se usó la variable diarrea, mediante la ENDES, se pregunta si en los 14 últimos días, el niño tuvo diarrea. Dicha variable toma dos valores: 0 si no lo tuvo y 1 en caso lo haya tenido.

b) Variables tratamiento

- Acceso al agua potable: Población (en este caso niños y niñas menores de 5 años) de la sierra del país que tienen una red de servicio de agua conectada con las tuberías de la vivienda.

- Acceso al alcantarillado: Población (en este caso niños y niñas de la sierra del Perú) que cuentan con el servicio de alcantarillado.

- Acceso a ambos (agua potable y alcantarillado)

Cada una de las variables tratamiento toman el valor de 1 si es que la vivienda tiene acceso a agua, a alcantarillado o a ambos respectivamente y el valor 0 , en caso contrario.

c) Variables de control: variables que incluyen características del hogar, de la vivienda, del niño y de la madre. Entre ellas si la vivienda tiene o no electricidad, televisor, número de miembros del hogar, educación de la madre, edad del niño en meses, si la vivienda es rica o no, edad del jefe de hogar, si la vivienda está ubicada en un área rural o no, si la madre trabaja o no.

1. Variable tratamiento: acceso al agua

El soporte común (muestra el grupo de tratamiento con el grupo de control) fue satisfecho con las variables de control $(X)$ : electricidad, TV, cantidad de niños menores de 5 años, rural, rico, si la madre tiene educación superior, si la madre trabaja y sexo del niño.

\section{Figura 1}

Luego que se tiene el soporte común, se pasa a medir el impacto que tiene el programa (acceso al agua) sobre la variable resultado. Aplicando tres métodos de emparejamiento obtenemos que: con el método del vecino más cercano, la probabilidad de tener diarrea en niños menores de cinco años disminuye en 1 pp.; con el método de radio, la probabilidad de tener diarrea se reduce en 2.6 pp.; y con el método de Kernel la probabilidad de prevalencia de enfermedades diarreicas baja en $0.8 \mathrm{pp}$.

\section{Tabla 3}

2. Variable tratamiento: acceso al alcantarillado El soporte común fue satisfecho con las variables de control $(\mathrm{X})$ : electricidad; TV; rural; rico; si la madre tiene educación primaria, secundaria o superior; si la madre trabaja y sexo del niño.
Figura 2

Aplicando tres métodos de emparejamiento obtenemos que: con el método del vecino más cercano, la probabilidad de tener diarrea en niños menores de cinco años aumenta en 0.1 pp.; con el método de estratificación, la probabilidad de tener diarrea se reduce en 0.3 pp.; y con el método de Kernel la probabilidad de prevalencia de enfermedades diarreicas baja en 1 pp.

Tabla 4

Se observa que, con el tratamiento de acceso a alcantarillado los signos bajo el emparejamiento del vecino más cercano y de estratificación salen contrarios a lo que se esperaría.

3. Variable tratamiento: acceso a ambos

El soporte común fue satisfecho con las variables de control $(\mathrm{X})$ : electricidad, $\mathrm{TV}$, rural, rico, si la madre tiene educación superior y sexo del niño.

Figura 3

Aplicando los tres métodos de emparejamiento obtenemos que: con el método del vecino más cercano, la probabilidad de tener diarrea en niños menores de cinco años disminuye en 1.8 pp.; con el método de radio, la probabilidad de tener diarrea se reduce en 3.2 pp.; y con el método de Kernel la probabilidad de prevalencia de enfermedades diarreicas baja en 3.7 pp.

Tabla 5

\section{Conclusiones}

Al evaluar el impacto por el método de PSM, se concluye que los servicios de acceso a agua potable y a alcantarillado sí tienen efectos positivos en la disminución de la probabilidad de prevalencia de enfermedades diarreicas para los niños ubicados en la sierra del Perú. Si la vivienda del niño tiene acceso al agua potable, la probabilidad de tener diarrea disminuye entre 1 punto porcentual (método del vecino más cercano) hasta 2.6 puntos porcentuales (aplicando el método del radio). En caso de que, el hogar del niño tenga acceso al servicio de alcantarillado, la probabilidad de sufrir episodios de diarrea disminuye en 1 pp (aplicando el método de Kernel). Mientras que, si la vivienda del niño tiene acceso a ambos (agua potable y alcantarillado), la probabilidad de tener diarrea disminuye entre 1 punto porcentual (método del vecino más cercano) hasta 3.7 puntos porcentuales (aplicando el método de Kernel). Se recomienda cubrir el acceso al agua potable y del alcantarillado en la sierra del país, para que las enfermedades diarreicas agudas disminuyan en los niños, esto contribuirá positivamente en la salud y en la calidad de vida de la población.

\section{Referencias}

Banco Interamericano de Desarrollo - BID (2021). Efectos del Programa de Agua Potable y Saneamiento para Pequeñas Localidades y Comunidades Rurales en Bolivia: resultados 
de la evaluacion intermedia https://publications.iadb.or g/es/efectos-del-programa-de-agua-potable-y-sane amiento-para-pequenas-localidades- $y$-comunidadesrurales

Carbajal, M. (2014)." Evaluacion del impacto del saneamiento en el Peru: efectos sobre la salud". Documento de trabajo 1. SUNASS

Carrasco-Choque, F. (2013). Impacto del consumo de agua potable sobre la salud de los hogares del Peru https: //dialnet.unirioja.es/servlet/articulo?codigo $=4729790$

Gonzalez, B., Velazquez, C. \& Epele, N. (2013). \El impacto de la red cloacal sobre la salud de los niños: un analisis a partir de la Encuesta Nacional de Nutricion y Salud" https://www.yumpu.com/es/document/read/4299002 0/el-impacto-de-la-red-cloacal-sobre-la-salud-de-los -ninos-un-analisis-a-partir-de-la-encuesta-nacionalde-nutricion-y-salud

INEI (2020). Peru: Formas de Acceso al Agua y Saneamiento Basico

INEI (2020a). Peru: Encuesta Demografica y de Salud FamiliarENDES 2019 https://www.inei.gob.pe/media/MenuRe cursivo/publicaciones_digitales/Est/Endes2019/

Lara, H. y Garcia-Salazar, E. (2019). Prevalencia de enfermedades asociadas al uso de agua contaminada en el Valle del Mezquital http://www.scielo.org.mx/pdf/edsc/v7n21/2007-8064edsc-7-21-91.pdf

Marino, D. (2020). Impacto de los servicios publicos de agua potable y alcantarillado sobre los logros educativos en el Peru https://repositorio.up.edu.pe/bitstream/handle/1 1354/2853/MarinoDiego_Tesis_maestria_2020.pdf?s equence $=1$

MVCS (2021a). Diagnostico de la situacion de brechas de infraestructura o de acceso a servicios publicos del sector vivienda, construccion y saneamiento. https://cdn.www. gob.pe/uploads/document/file/1552487/Diagnostico $\% 20 \mathrm{de} \% 20$ Brechas.pdf

MVCS (2021b). Evaluacion de impacto del Programa Nacional de Saneamiento Urbano en Salud y Mortalidad Infantil https://cdn.www.gob.pe/uploads/document/file/189 $1100 /$ Evaluaci\%C3\%B3n\%20de $\% 20$ Impacto\%20del\% 20PNSU\%20en $\% 20$ Salud $\% 20 y \% 20$ Mortalidad\%20Inf antil.pdf

OMS (2017) Enfermedades diarreicas https://www.who.int/es /news-room/fact-sheets/detail/diarrhoeal-disease

OMS/UNICEF (2021). Progres son household drinking water, sanitation and higiene 2000-2020: Five years into the SDGs

Rosenbaum, P. y Rubin, D.(1983). The central role of the propensity score in observational studies for causal effects https://academic.oup.com/biomet/article/70/1/41/24 0879

Sotelo, M (2016). Impacto del acceso a los servicios de agua y saneamiento sobre la desnutricion cronica infantil: evidencia del Peru https://tesis.pucp.edu.pe/reposi torio/handle/20.500.12404/9841

UNESCO (2019) Informe Mundial de Naciones Unidas sobre el Desarrollo de los Recursos Hıdricos 2019 https: //www.acnur.org/5c93e4c34.pdf
UNESCO (2020). Agua y Cambio climatico https://es.unesco. org/themes/water-security/wwap/wwdr/2020\#downl oad 


\section{Lista de tablas}

Tabla 1: Indicadores de Brecha de los Servicios de acceso al agua potable y de alcantarillado en el ámbito rural

\begin{tabular}{|c|c|c|c|c|c|c|c|}
\hline & & & & \multicolumn{4}{|c|}{ Línea Base 2019} \\
\hline Servicio Público & Nombre de indicador & $\begin{array}{l}\text { Tipo de } \\
\text { indicador }\end{array}$ & $\begin{array}{l}\text { Línea base } 2018 \\
\text { Brecha }(\%)\end{array}$ & $\begin{array}{l}\text { Deman- } \\
\text { dado }\end{array}$ & $\begin{array}{l}\text { Implemen- } \\
\text { tado }\end{array}$ & $\begin{array}{c}\text { Por } \\
\text { Implementar }\end{array}$ & $\begin{array}{c}\text { Brecha } \\
(\%)\end{array}$ \\
\hline \multirow[t]{2}{*}{ Agua Potable } & $\begin{array}{l}\text { Porcentaje de la población } \\
\text { rural sin acceso al servicio de } \\
\text { agua potable mediante red o } \\
\text { pileta publica }\end{array}$ & Cobertura & $34,00 \%$ & 7605868 & 4556412 & 3049456 & $40,09 \%$ \\
\hline & $\begin{array}{l}\text { Porcentaje de viviendas ru- } \\
\text { rales con servicio de agua con } \\
\text { cloro residual menor al límite } \\
\text { permisible }(0,5 \mathrm{mg} / \mathrm{l})\end{array}$ & Calidad & $97,44 \%$ & 2068678 & 78779 & 1989900 & $96,19 \%$ \\
\hline Alcantarillado & $\begin{array}{l}\text { Porcentaje de la población } \\
\text { rural sin acceso al servicio de } \\
\text { alcantarillado u otras formas } \\
\text { de disposición sanitaria de } \\
\text { excretas }\end{array}$ & Cobertura & $54,32 \%$ & 7605868 & 3248823 & 4357045 & $57,29 \%$ \\
\hline
\end{tabular}

Fuente: Ministerio de Vivienda, Construcción y Saneamiento Elaboración: Propia

Tabla 2: Departamentos con mayor brecha de cobertura del Servicio de Agua Potable-Rural

\begin{tabular}{|c|c|c|c|c|}
\cline { 2 - 5 } \multicolumn{1}{c|}{} & \multicolumn{4}{|c|}{ Línea de Base-2019 } \\
\hline Departamento & Total Población Rural & Población con acceso & Población sin acceso & Brecha (\%) \\
\hline Perú & 7.605 .868 & 4.556 .412 & 3.049 .456 & $40,09 \%$ \\
\hline Loreto & 350.437 & 68.697 & 281.740 & $80,40 \%$ \\
\hline Ucayali & 127.526 & 36.231 & 91.295 & $71,59 \%$ \\
\hline Madre de Dios & 43.514 & 17.260 & 26.254 & $60,33 \%$ \\
\hline Puno & 602.469 & 257.543 & 344.926 & $57,25 \%$ \\
\hline Tacna & 46.215 & 19.923 & 26.292 & $56,89 \%$ \\
\hline Junín & 434.164 & 208.096 & 226.068 & $52,07 \%$ \\
\hline Moquegua & 32.110 & 16.319 & 15.791 & $49,18 \%$ \\
\hline Pasco & 141.471 & 72.453 & 69.018 & $48,79 \%$ \\
\hline Huánuco & 391.405 & 209.336 & 182.069 & $46,52 \%$ \\
\hline Piura & 543.169 & 291.457 & 251.712 & $46,34 \%$ \\
\hline
\end{tabular}

Fuente: Ministerio de Vivienda, Construcción y Saneamiento 
Tabla 3: Métodos de Emparejamiento - Acceso a agua potable

\begin{tabular}{|c|c|c|c|}
\hline Método de Emparejamiento & Vecino más cercano & Radio & Kernel \\
\hline ATT & $-0,01$ & $-0,026$ & $-0,008$ \\
\hline
\end{tabular}

Tabla 4: Métodos de Emparejamiento - Acceso a alcantarillado

\begin{tabular}{|c|c|c|c|}
\hline Método de Emparejamiento & Vecino más cercano & Radio & Kernel \\
\hline ATT & 0,001 & 0,003 & $-0,01$ \\
\hline
\end{tabular}

Tabla 5: Métodos de Emparejamiento - Acceso a agua potable y alcantarillado

\begin{tabular}{|c|c|c|c|}
\hline Método de Emparejamiento & Vecino más cercano & Radio & Kernel \\
\hline ATT & $-0,018$ & $-0,032$ & $-0,037$ \\
\hline
\end{tabular}




\section{Lista de figuras}

Figura 1: Soporte Común - Acceso a Agua potable

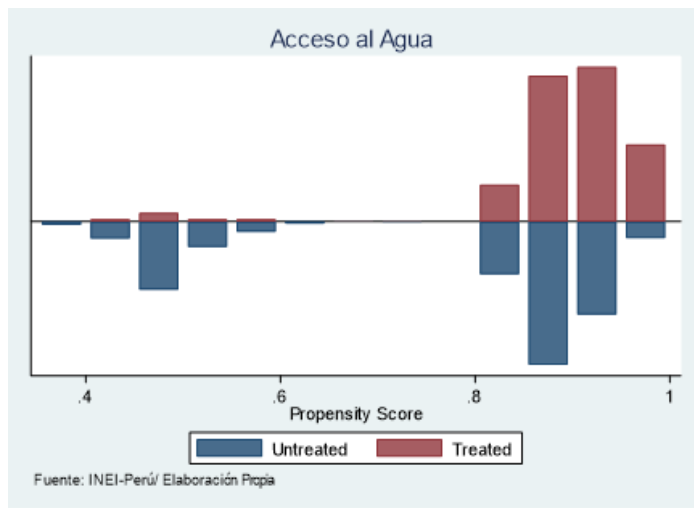

Figura 2: Soporte Común - Acceso a alcantarillado

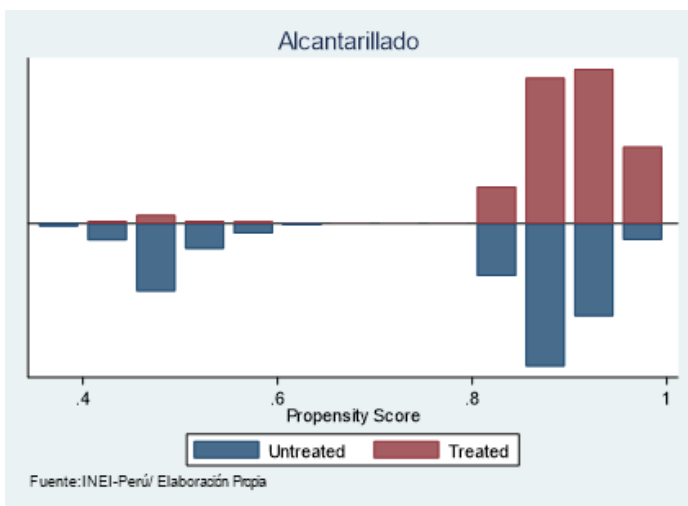

Figura 3: Soporte común - Acceso a agua potable y alcantarillado

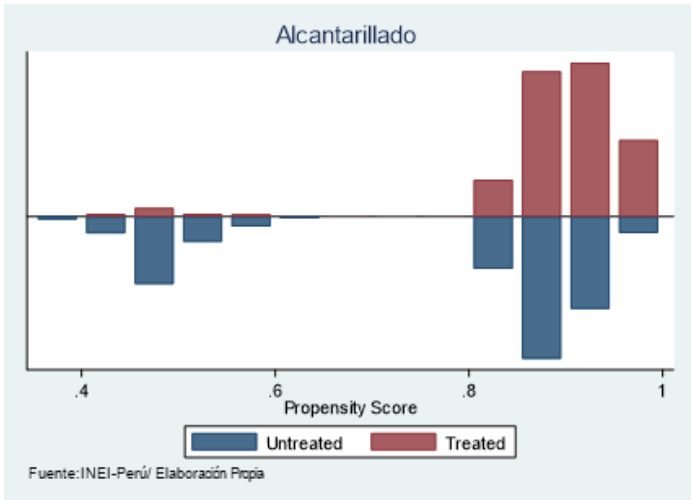

